\title{
Editorial \\ Prevention of Early-onset GBS Sepsis: Evaluation of a Changing Paradigm
}

\section{Beverly S. Brozanski, MD}

Journal of Perinatology (2003) 23, 263-264. doi:10.1038/sj.jp.7210940

Prevention of neonatal sepsis caused by group B streptococcus (GBS) is the goal of current, prophylactic strategies. A recent population-based surveillance study has shown that antepartum screening for GBS was 50\% more effective than the risk-based approach at preventing perinatal GBS. ${ }^{1}$ This study led to the updated guidelines published by the $\mathrm{CDC}$, which provide recommendation for universal prenatal culture-based screening for GBS colonization as well as instruction on specimen collection, culture processing, and susceptibility testing and prophylaxis regimens for women with penicillin allergy. ${ }^{2}$

Coincident with active prevention efforts in the 1990's, the incidence of early-onset GBS decreased from two to three cases per 1000 live births to 0.5 cases per 1000 live births (1). In this issue of the Journal of Perinatology, studies by Riley et al. ${ }^{3}$ as well as Pinto et al. ${ }^{4}$ evaluated the effectiveness of and residual problems associated with the culture-based and risk-based GBS prevention strategies.

Riley et al. ${ }^{3}$ examined compliance with intrapartum antibiotic prophylaxis, which they found to be dependent on the practice setting and the prevention strategy employed. Several of the problems they identified will no longer be an issue when birthing centers replace the risk-based strategy with the culture-based GBS prevention strategy. In Riley's study, for those women managed with the culture-based strategy, almost $10 \%$ failed to have documentation of GBS screening-culture results at the time of delivery and up to $6 \%$ of the GBS screening cultures were obtained in the first trimester. Of women whose screening cultures were positive for GBS, 30 to 46\% were treated with antibiotics for less than the optimal 4 hours before delivery. In Riley's estimation, 50\% of those women could have received antibiotics at least 4 hours before delivery. These authors recommended that patient education about GBS screening-culture results and strict attention to maternal GBS culture status upon presentation in labor would improve compliance with antibiotic administration within 4 hours of delivery.

Pinto et al. ${ }^{4}$ reviewed 94 cases of early-onset GBS admitted to two regional level III NICUs from $07 / 92$ through $12 / 01$. Of these

Department of Pediatrics and Obstetrics, Gynecology and Reproductive Sciences University of Pittsburgh, USA.

Address correspondence and reprint requests to Beverly S. Brozanski, MD, Department of Pediatrics and Obstetrics, Gynecology and Reproductive Sciences University of Pittsburgh, USA. infants, $50(53 \%)$ were born to women whose GBS colonization status was (i) unknown and who had no identified risk factors or (ii) whose GBS screening culture was reported as negative. The majority of missed opportunities for appropriate administration of intrapartum antibiotics were attributed to: failure to perform or faulty performance of cultures, unreliable culture results, and antibiotic administration for less than 4 hours before delivery.

Few studies to date have evaluated implementation and efficacy of CDC protocol for management of infants born to women colonized with GBS. Pinto recommends further evaluation of adjunctive postnatal prophylaxis, which in reality would represent early treatment. ${ }^{4}$ In his cohort, such postnatal "prophylaxis" would not have been effective, as disease presented clinically at $2.1 \pm 1.3$ hours (mean $\pm \mathrm{SD}$ ) of life. The revised $\mathrm{CDC}$ protocol recommended complete blood cell count, blood culture and a 48hour hospital evaluation of all asymptomatic infants beyond 34 weeks gestation that were born to women receiving intrapartum antibiotics less than 4 hours before delivery. ${ }^{2}$ Unfortunately, the sensitivity, specificity and predictive values of a single blood count for infection are variable. ${ }^{5}$ Observation for a minimum of 48 hours has been recommended because of concern that intrapartum antibiotics may alter the presentation of newborn sepsis. Recent data do not support this supposition. Bromberger et al. reported on 172 term infants with blood cultures positive for GBS identified from a population of 277,912 live-born infants. Of the infants who developed clinical signs of infection, $95 \%$ presented within 24 hours after birth. An additional $3.7 \%$ of infants became symptomatic within 48 hours and only 1.2\% developed symptoms after 48 hours of life. These authors concluded that neither obstetrical risk factors nor intrapartum antibiotics altered the spectrum and onset of clinical signs of GBS infection.

At Magee-Womens Hospital, we monitored the effectiveness of a culture-based GBS prevention strategy from October 1995 through December 2002. GBS-screening culture results were documented for over $96 \%$ of deliveries at $\geq 36$ weeks gestation. In a subset of these women eligible for intrapartum prophylaxis, 94\% received antibiotics before to delivery. ${ }^{7}$ The incidence of early-onset GBS disease during the 7-year period was 0.2 per 1000 (11 cases in 54,908 live births). Five of these infants were born to women with negative antepartum GBS cultures (one treated intrapartum for chorioamnionitis), three ( $<30$ weeks gestation) were born to women with unknown GBS colonization, and 3 were born to women known to be colonized with GBS (two with precipitous delivery within three hours of presentation and one who received antibiotics 5 hours prior to delivery). All infants presented before 16 hours of life. Successful implementation of a culture-based GBS 
prevention protocol, in our institution, was associated with persistently low occurrence of early-onset GBS. In our hospital, we recommend a minimum of 36 hours of observation for all term or near-term asymptomatic infants who are born to women known to be GBS carriers or whose carrier status is unknown but who have a risk factor(s) for invasive GBS disease. Routine laboratory testing is not performed. Infants manifesting any sign of systemic infection are transferred to the neonatal intensive care unit for complete evaluation and treatment.

Infants born to women with signs and symptoms of chorioamnionitis, regardless of GBS colonization status, are at increased risk for sepsis. ${ }^{5}$ While close observation of all infants born to women who meet clinical criteria for chorioamnionitis is warranted, antibiotic treatment of asymptomatic infants whose mothers have chorioamnionitis is not supported by available data. In our hospital, laboratory testing and treatment for presumptive infection is not routine, but these infants do not qualify for early discharge.

Implementation of a GBS prevention strategy is not limited by clinical setting. Effective compliance strategies have been described in large university-affiliated centers, ${ }^{7}$ community hospitals without academic affiliation ${ }^{8}$ and in health maintenance organizations. ${ }^{9}$ Systemwide factors that contributed to successful implementation of a GBS prevention protocol included: physician-leaders as catalysts for protocol presentation and implementation, provider education through meetings and mass mailings, and preprinted order forms that specifically address GBS colonization and antibiotic prophylaxis. However, despite our best attempts, implementation of intrapartum prophylaxis will fail to occur in some cases. Development of a rapid test for GBS that can be performed in a timely fashion and is linked to immediate administration of antibiotics may improve intrapartum prophylaxis. Nonetheless, even with adequate intrapartum prophylaxis, cases of early-onset GBS will continue to be reported. Additional data must be collected before routine evaluation for infection and empiric antibiotic treatment in asymptomatic infants is considered "standard of care" or "best practice". Methods to diagnose presumptive infection with PCR-based tools may identify those infants with blood-borne infection before symptoms are apparent. We look forward to the results of ongoing vaccine trials, which may facilitate a decrease in maternal GBS colonization and concomitant reduction in early-onset GBS disease. As guidelines aimed at the most effective means of reducing early-onset GBS sepsis continue to be refined, it is clear that we need collaborative efforts to monitor and report on those management strategies.

\section{References}

1. Schrag SJ, Zell ER, Lynfield R, et al. A population-based comparison of strategies to prevent early-onset group B streptococcal disease in neonates. N Engl J Med 2002;347:233-9.

2. Centers for Disease Control and Prevention. Prevention of Perinatal Group B Streptococcal Disease. MMWR 2002;51(RR-11):1-22.

3. Riley L, Apollon K, Haider S, et al. "Real world" compliance with strategies to prevent early onset group B streptococcal disease. J Perinatol 2003;23:272-7.

4. Pinto NM, Soskolne El, Pearlman MD, Faix RG. Neonatal early-onset group B streptococcal disease in the era of intrapartum chemoprophylaxis: residual problems. J Perinatol 2003;23:265-71.

5. Gerdes JS. Clinicopathologic approach to diagnosis of neonatal sepsis. Clin Perinatol 1991;8:361-81.

6. Bromberger P, Lawrence JM, Braun D, Saunders B, Contreras R, Petitti DB. The influence of intrapartum antibiotics on the clinical spectrum of earlyonset group B streptococcal infection in term infants. Pediatrics 2000;106:244-50.

7. Brozanski BS, Jones JG, Krohn MA, Sweet RL. Effect of a screening-based prevention policy on prevalence of early-onset group B Streptococcal sepsis. Obstet Gynecol 2000;95:496-501.

8. Clemens CJ, Gable EK. The development of a group B streptococcus prevention policy at a community hospital. J Perinatol 2002;22:523-5.

9. Davis RL, Hasselquist MB, Cardenas V, et al. Introduction of the new centers for disease control and prevention of group B streptococcal prevention guideline at a Large West Coast Health Maintenance Organization. Am J Obstet Gynecol 2001;184:603-10. 